\title{
Visualization of CombiHIVvac Vaccine Particles Using Electron Microscopy
}

\author{
Larisa I. Karpenko, Leonid R. Lebedev, Sergei I. Bazhan, Denis V. Korneev, \\ Boris B. Zaitsev, and Alexander A. llyichev
}

Keywords: HIV vaccine, polyepitope immunogens, DNA vaccine, artificial proteins, self-assemble microparticles, phase I clinical trials

A CANDIDATE VACCINE CombiHIVvac is developed; presently the clinical phase I trial has been completed successfully. CombiHIVvac combines the conserved polyepitope immunogens approaches in a novel self-adjuvanted microparticle concept. The artificial TBI (T cell and B cell immunogen) polypeptide used in the vaccine comprises epitopes from Env and Gag. The polypeptide is conjugated to dextran and mixed with DNA, which leads to formation of microparticles presenting TBI on the surface and containing the DNA inside. The DNA (pcDNA-TCI) enclosed in the microparticles codes for the TCI (T cell immunogen) polypeptide, which contains $\mathrm{CD}^{+}$and $\mathrm{CD}^{+}$epitopes from Env, Gag, Pol, and Nef conserved among HIV subtypes A, B, and C. ${ }^{1,2}$ The proposed technique enables the vaccine components to combine into particles on the principle of self-assembly (Fig. 1A).

The conjugate molecule consists of 1 dextran molecule, 1 protein TBI molecule, and 10-15 spermidine molecules.
Positively charged spermidine provides binding of the conjugate dextran/protein TBI with negatively charged DNAvaccine promoting formation of particles on the self-assembly principle.

According to our estimation, the plasmid pcDNA-TCI $(6,583 \mathrm{bp})$ is able to present about 100-110 dextran $(60 \mathrm{kDa})$ molecules on its surfaces. For vaccine assembly, pcDNA-TCI was added to conjugate in the proportion of 1 DNA molecule:120 conjugate molecules. To prove that CombiHIVvac has actually the form of particles, we used gel filtration chromatography and atomic force microscopy. ${ }^{2}$ During sepharose CL-2B gel filtration, the vaccine was eluted in the volume, corresponding to 12-14 MDa size material.

Experimental visualization of a theoretically predicted formation of artificial microparticles was performed with transmission electron microscopy with negative staining $(1 \%$
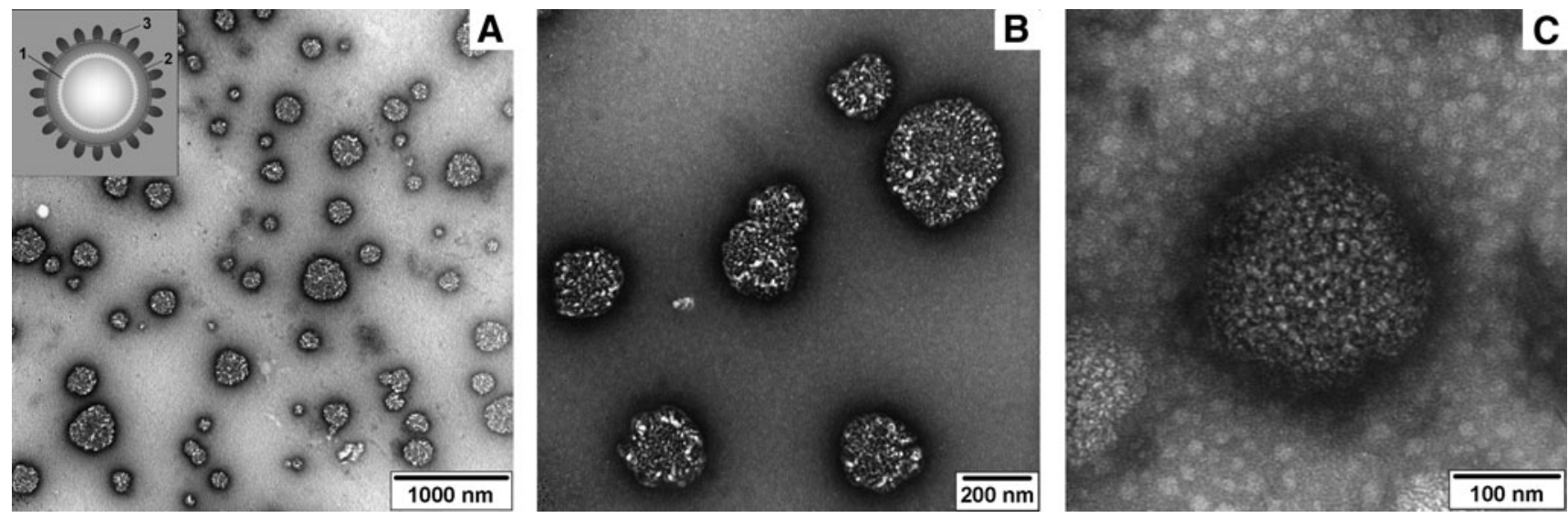

FIG. 1. Transmission electron microscopy images of CombiHIVvac microparticles with different magnification. (A) Scale bar $1,000 \mathrm{~nm}$, the inset in the left upper corner is a scheme of a CombiHIVvac particle (1-pcDNA-TCI, 2-spermidine/ dextran, 3-TBI); (B) Scale bar $200 \mathrm{~nm}$; (C) Scale bar $100 \mathrm{~nm}$.

State Research Center of Virology and Biotechnology "Vector," Koltsovo, Russia.

(C) Larisa I. Karpenko, et al., 2017; Published by Mary Ann Liebert, Inc. This Open Access article is distributed under the terms of the Creative Commons Attribution Noncommercial License (http://creativecommons.org/licenses/by-nc/4.0/) which permits any noncommercial use, distribution, and reproduction in any medium, provided the original author(s) and the source are credited. 
aqueous uranyl acetate solution). JEM 1400 electronic microscope (Jeol, Japan) with accelerating voltage $80 \mathrm{kV}$ was used.

The obtained images demonstrate particles $50-250 \mathrm{~nm}$ in diameter (Fig. 1A-C). The morphological similarity of these objects to native virus particles is obvious.

Previously we showed that by combining two immunogens (TBI and TCI) in one construct, significant enhancement of HIV-specific B cell response was observed. ${ }^{3}$ In our opinion, the formation of such particles plays a critical role in the registered effect. CombiHIVvac particles enable more effective absorption by antigen-presenting cells than individual molecules. As TBI protein is fixed on the particle surface and is represented in multiple copies, this provides multiple enhancement of vaccine antigenicity. Besides, pcDNA-TCI enclosed in the vaccine structure is more protected against degradation by DNase I than free pcDNA-TCI, as it was previously demonstrated, ${ }^{4}$ resulting in prolongation of DNAvaccine presence in organism. Finally, the presence of MHC class II restricted $\mathrm{CD}^{+}{ }^{+} \mathrm{T}$-helper epitopes in the protein $\mathrm{TCI}$ may be the main reason underlying the increased synthesis of antibodies to TBI protein because of a CD4-mediated stimulation of $\mathrm{B}$ cell proliferation and differentiation.

Thus, the obtained high-resolution images of CombiHIVvac artificial microparticles show that vaccine structure mimics the size and organization of native viruses and yield insights into how this structure relates to high immunogenicity of the vaccine.

\section{Acknowledgment}

A part of the study concerning the electron microscopy of CombiHIVvac was supported by the Russian Science Foundation (no. 14-14-00660 grant).

\section{Author Disclosure Statement}

No competing financial interests exist.

\section{References}

1. Bazhan SI, Belavin PA, Seregin SV, et al:: Designing and engineering of DNA-vaccine construction encoding multiple CTL-epitopes of major HIV-1 antigens. Vaccine 2004;22: 1672-1682.

2. Karpenko LI, Ilyichev AA, Eroshkin AM, et al: : Combined virus-like particle-based polyepitope DNA/protein HIV-1 vaccine. Design, immunogenicity and toxicity studies. Vaccine 2007;25:4312-4323.

3. Bazhan SI, Karpenko LI, Lebedev LR, et al:: A synergistic effect of a combined bivalent DNA-protein anti-HIV-I vaccine containing multiple T- and B-cell epitopes of HIV-1 proteins. Mol Immunol 2008;45:661-669.

4. Karpenko LI, Nekrasova NA, Ilyichev AA, et al:: Comparative analysis using a mouse model of the immunogenicity of artificial VLP and attenuated Salmonella strain carrying a DNA-vaccine encoding HIV-1 polyepitope CTLimmunogen. Vaccine 2004;22:1692-1699.

Address correspondence to:

Larisa I. Karpenko

State Research Center of Virology and Biotechnology "Vector"

Novosibirsk Region

Koltsovo 630559

Russia

E-mail: karpenko@vector.nsc.ru 